\title{
Metanálise sobre o Uso de Glicocorticóides Pré-Operatório para Redução do Risco de Complicações após Esofagectomia por Carcinoma do Esôfago*
}

\author{
Use of Preoperative Glucocorticoid to Risk Reduction of Complications \\ after Esophagectomy by Esophagus Carcinoma: Meta-Analysis.
}

Antônio Marcos Raimondi ${ }^{1}$

\section{RESUMO}

JUSTIFICATIVA E OBJETIVOS: O emprego de glicocorticóides tem sido proposto para reduzir a morbidade após intervenções cirúrgicas, porém, não é largamente utilizado antes da ressecção do esôfago porque não existem conhecimentos suficientes sobre a sua eficácia. O objetivo do trabalho foi verificar os efeitos da administração pré-operatória de glicocorticóides, em pacientes submetidos à esofagectomia por carcinoma de esôfago. MÉTODO: As fontes utilizadas foram: MEDLINE, EMBASE, Cancerlit, SCIELO, Base de Dados de Ensaios Clínicos Controlados da Colaboração Cochrane e busca manual de referências. O término da pesquisa ocorreu em dezembro de 2005. Foram pesquisados estudos aleatórios de pacientes com carcinoma esofágico, em que se compararam glicocorticóides com placebo, administrados antes das esofagectomias. Os dados foram coletados pelos mesmos revisores e a qualidade dos estudos foi avaliada usando-se o escore de Jadad. A metanálise foi realizada utilizando-se a razão de chances - odds ratio- (intervalos de confiança - IC - de 95\%) entre tratamento e placebo e um modelo de risco relativo baseado no teorema de Bayes.

1. Mestre em Medicina, Médico Intensivista da Unidade de Terapia Intensiva de Neurocirurgia - Hospital das Clínicas da Faculdade de Medicina de Ribeirão Preto da Universidade de São Paulo, Ribeirão Preto, SP.

*Recebido do Hospital das Clínicas de Ribeirão Preto, Ribeirão Preto, SP

Apresentado em 12 de julho de 2006

Aceito para publicação em 06 de dezembro de 2006

Endereço para correspondência:

Hospital das Clínicas de Ribeirão Preto

Secretaria do Departamento de Cirurgia - $9^{\circ}$ andar.

14048-900 Ribeirão Preto, SP.

Fone: 16 - 3602-2592

E-mail: raimond11@terra.com.br

(C)Associação de Medicina Intensiva Brasileira, 2006
RESULTADOS: Quatro estudos envolvendo 169 pacientes foram localizados. Não ocorreram diferenças na mortalidade pós-operatória e nas incidências de deiscência de anastomose e insuficiências renal e hepática entre glicocorticóides e placebo. Houve decréscimo na incidência de complicações respiratórias pós-operatórias $(95 \% \mathrm{IC}=0,09-0,46)$, sepse $(95 \% \mathrm{IC}=0,10-0,81)$ e no total de complicações pós-operatórias $(95 \%$ IC = 0,06-0,23) no grupo tratado com glicocorticóides.

CONCLUSÕES: A administração pré-operatória de glicocorticóides foi associada com um decréscimo nas complicações pós-operatórias.

Unitermos: Esofagectomia, Falência de múltiplos órgãos, Literatura de revisão, Metanálise, Metilprednisolona.

\section{SUMMARY}

BACKGROUND AND OBJECTIVES: Preoperative glucocorticoid administration has been proposed for reducing postoperative morbidity. This is not widely used before esophageal resection because of incomplete knowledge regarding its effectiveness. The aim here was to assess the effects of preoperative glucocorticoid administration in adults undergoing esophageal resection for esophageal carcinoma.

METHODS: Studies were identified by searching the Cochrane Controlled Trials Register, MEDLINE, EMBASE, CancerLit, SCIELO and Cochrane Library, and by manual searching from relevant articles. The last search for clinical trials for this systematic review was performed in December 2005. This review included randomized studies of patients with potentially resectable carcinomas of the esophagus that compared preoperative glucocorticoid administration with placebo. Data were extracted by the reviewer, and the trial quality was assessed using Jadad scoring. Odds ratio with 95\% 
confidence limits and bayesian relative risk were used to assess the significance of the difference between the treatment arms.

RESULTS: Four randomized trials involving 169 patients were found. There were no differences in postoperative mortality, anastomotic leakage, hepatic and renal failure between the glucocorticoid and placebo groups. There were fewer postoperative respiratory complications $(95 \% \mathrm{Cl}=0.09-0.46)$, sepsis $(95 \% \mathrm{Cl}=0.10-0.81)$, and total postoperative complications $(95 \% \mathrm{Cl}=0.06-0.23)$ with preoperative glucocorticoid administration.

CONCLUSIONS: Prophylactic administration of glucocorticoids is associated with decreased postoperative complications.

Key Words: Esophagectomy, Meta-analysis, Methylprednisolone, Multiple organ failure, Review literature.

\section{INTRODUÇÃO}

As cirurgias de grande porte induzem uma resposta inflamatória, como a elevação dos níveis dos hormônios adrenocorticotrópicos e reagentes da fase aguda $^{1-3}$. Esta resposta inflamatória intensa está relacionada com o desenvolvimento de complicações pós-operatórias graves ${ }^{2,3}$. A esofagectomia é um dos procedimentos cirúrgicos mais invasivas do trato gastrintestinal e apresenta elevada morbidade devido às complicações pós-operatórias ${ }^{4-7}$. Estas complicações estão relacionadas com a intensa resposta inflamatória sistêmica provocada pela invasão simultânea das estruturas do abdômen, mediastino e região cervical durante $o$ ato operatório, em pacientes previamente debilitados por afecções esofágicas benignas e malignas ${ }^{4}$. Particularmente, a insuficiência respiratória pode ser fatal e a hipoxemia pós-operatória é a causa mais importante de deiscência da anastomose ${ }^{2,3}$.

Os glicocorticóides podem ser efetivos na redução da resposta inflamatória secundária à sepse e outros estados relacionados ao estresse orgânico ${ }^{1,8,9}$. Estudos relataram que a administração pré-operatória de metilprednisolona reduz a resposta metabólica, protege contra a elevação dos níveis de citocinas pró-inflamatórias e assegura oxigenação adequada após esofagectomia ${ }^{2,3,10-13}$. É descrito que os glicocorticóides são importantes na regulação do sistema nervoso simpático e da medula adrenal ${ }^{3,8,9}$. A redução da norepinefrina circulante parece se originar dos neurônios simpáticos mais do que da adrenal ${ }^{3,8,9}$. Os glicocorticóides são conhecidos como agentes antiinflamatórios potentes pela estabilização da membrana lisossômica ${ }^{3,8,9}$.
Os corticosteróides primariamente inibem fatores de transcrição como o fator kB nuclear e AP-1. Assim, uma vez que a produção do RNA mensageiro se inicie, os corticosteróides seriam inefetivos.

Quando há poucos ensaios clínicos sobre um tratamento, a metanálise é o melhor instrumento para verificar a magnitude de seus efeitos e se os resultados da intervenção não são devidos ao acaso ${ }^{14-20}$. É importante salientar que, excluídos vícios metodológicos, como toda inferência estatística, a metanálise envolve uma estimativa de amostras conhecidas extrapoladas para uma população desconhecida. Deste modo, nível de significância, intervalo de confiança e probabilidades a priori e a posteriori são métodos para lidar com esta incerteza ${ }^{21-27}$.

O emprego de glicocorticóides foi proposto para reduzir a morbidade após procedimentos cirúrgicos, porém não é largamente utilizado na ressecção do esôfago, devido ao fato de não haver ainda conhecimentos suficientes sobre sua eficácia e segurança.

O objetivo deste estudo foi verificar os efeitos do uso pré-operatório de glicocorticóides nos pacientes submetidos à esofagectomia por carcinoma de esôfago. Os desfechos selecionados neste estudo foram relacionados com a resposta inflamatória sistêmica e complicações pós-operatórias.

\section{MÉTODO}

Esta revisão sistemática incluiu estudos aleatórios de pacientes com carcinoma de esôfago potencialmente ressecável de qualquer tipo histológico, que compararam a administração pré-operatória de glicocorticóides com placebo.

Os estudos foram identificados por meio da pesquisa nas seguintes bases de dados: registro de ensaios controlados da colaboração Cochrane, MEDLINE (19662005), EMBASE (1988 - 2004), CancerLit (1993 - 2004), SCIELO (1993-2004) e Cochrane. Foram procurados estudos que pudessem ser considerados aleatórios e com sigilo de alocação adequado a partir de 1995. Adicionalmente, foram realizadas pesquisas manuais de artigos relevantes, de comunicações pessoais, contato com autores e fabricantes.

A seguinte estratégia de busca foi elaborada usado uma combinação de palavras-chaves e de textos relacionadas ao uso de glicocorticóides e do tratamento do carcinoma de esôfago: randomized controlled trial. pt; randomized controlled trials/random allocation/double-blind method; single-blind method; 1 or 2 or 3 or 4 or 5; animal; human; 7 not (7 and 8); 6 not 9; clinical 
trial.pt; exp clinical trials; (clin\$ adj3 trial\$).ab,ti; ((singl\$ or doub/\$ or treb\$ or trip\$) adj3 (blind\$ or mask\$)). ab,ti; placebos; placebo\$.ab,ti; random.ab,ti; research design; 11 or 12 or 13 or 14 or 15 or 16 or 17 or $18 ; 19$ not 9; 20 not 10; comparative study; exp evaluation studies; follow-up studies; prospective studies; (contro/\$ or prospectiv\$ or volunteer\$).ab,ti; 21 or 22 or 23 or 24 or 25; 26 not 9; 28 not (10 or 21); exp esophageal neoplasms; exp esophagus; esophag\$.tw; (esophag\$ adj2 neoplas\$).tw; (oesophag\$ adj2 neoplas\$).tw; (esophag\$ adj2 cancer).tw; (oesophag\$ adj2 cancer).tw; exp combined modality therapy; exp drug therapy combination; exp chemotherapy adjuvant; exp radiotherapy adjuvant; glicocorticoid.tw; methylprednisolone.tw; corticosteroid.tw; complication.tw; sepsis.tw; respiratory.tw; hepatic.tw; renal.tw; death.tw; sirs.tw; lung.tw; mortality. tw; organ failure.tw; exp treatment outcome.

Os dados foram extraídos pelo revisor e a qualidade dos ensaios clínicos foi avaliada utilizando o escore de
Jadad $^{24}$. O principal critério de inclusão de cada estudo foi a existência de processo de classificação com sigilo de alocação adequado. O sigilo de alocação foi considerado adequado quando foi relatado detalhadamente ou mencionado no texto que o estudo foi aleatório, indicando que a alocação pareceu ser correta.

Foram encontrados 172 artigos, dos quais seis foram considerados estudos aleatórios e com sigilo de alocação adequado. Destes, foram excluídos dois artigos que não apresentavam os desfechos de interesse para esta metanálise. Foram incluídos quatro estudos ${ }^{2,10-12}$, com o total de 169 pacientes (Tabelas 1 e 2). Destes, 82 receberam metilprednisolona (grupo tratamento) e 87 receberam solução fisiológica (grupo placebo) durante a indução anestésica.

A quantificação do viés de publicação foi realizada por meio do teste de assimetria aplicado ao gráfico do funil (funnel plot), que relaciona a razão de chances com o tamanho das amostras (Tabela 3). Nesta análise, quan-

Tabela 1 - Ensaios Clínicos Incluídos na Revisão Sistemática

\begin{tabular}{lcccc}
\hline Autores & Escore de Jadad & Amostra (N) & Intervenção (N) & Placebo (N) \\
\hline Sato e col. ${ }^{12^{*}}$ & 5 & 66 & 33 & 33 \\
Matsutani e col. ${ }^{1{ }^{*}}$ & 3 & 33 & 14 & 19 \\
Takeda e col. ${ }^{2 \dagger}$ & 3 & 30 & 15 & 15 \\
Sayama e col. ${ }^{10 \ddagger}$ & 3 & 40 & 20 & 20 \\
Total & & 169 & 82 & 87 \\
\hline
\end{tabular}

*Metilprednisolona - 10 mg/kg antes da indução anestésica.

†Metilprednisolona - $30 \mathrm{mg} / \mathrm{kg}$ antes da indução anestésica.

¥Metilprednisolona - 250 mg antes da indução anestésica.

Tabela 2 - Características dos Grupos Tratamento e Placebo

\begin{tabular}{|c|c|c|c|c|c|c|c|c|}
\hline \multirow[t]{2}{*}{ Características } & \multicolumn{4}{|c|}{ Grupo Tratamento } & \multicolumn{4}{|c|}{ Grupo Placebo } \\
\hline & $\begin{array}{c}\text { Matsutani e col. } \\
\mathrm{N}=14\end{array}$ & $\begin{array}{c}\text { Sato e col. } \\
\mathrm{N}=33\end{array}$ & $\begin{array}{c}\text { Takeda e col. } \\
N=15\end{array}$ & $\begin{array}{c}\text { Sayama e col. } \\
N=33\end{array}$ & $\begin{array}{l}\text { Matsutani e col. } \\
\mathrm{N}=19\end{array}$ & $\begin{array}{c}\text { Sato e col. } \\
\quad N=33\end{array}$ & $\begin{array}{c}\text { Takeda e col. } \\
\mathrm{N}=15\end{array}$ & $\begin{array}{c}\text { Sayama e col. } \\
\mathrm{N}=33\end{array}$ \\
\hline $\begin{array}{l}\text { Duração da Ci- } \\
\text { rurgia }(\min )^{\star}\end{array}$ & $431 \pm 60$ & $330 \pm 33$ & $427 \pm 65$ & $415 \pm 40$ & $431 \pm 51$ & $339 \pm 49$ & $485 \pm 105$ & $435 \pm 47$ \\
\hline $\begin{array}{l}\text { Hemorragia intra- } \\
\text { operatória }(\mathrm{g})^{*}\end{array}$ & $795 \pm 512$ & $696 \pm 314$ & $853 \pm 474$ & $793 \pm 412$ & $785 \pm 377$ & $647 \pm 286$ & $960 \pm 533$ & $805 \pm 355$ \\
\hline Idade (anos)* & $64 \pm 7$ & $62 \pm 8$ & $63 \pm 7$ & $63 \pm 7$ & $64 \pm 7$ & $64 \pm 7$ & $63 \pm 10$ & $64 \pm 7$ \\
\hline Sexo Masculino & 12 & 29 & 13 & 28 & 12 & 31 & 14 & 29 \\
\hline
\end{tabular}

${ }^{*}$ Dados expressos em Média \pm DP

Tabela 3 - Resumo da Análise do Viés de Publicação

\begin{tabular}{lccc}
\hline \multirow{2}{*}{ Desfecho Pós-Operatório } & \multirow{2}{*}{ Participantes $(\mathrm{N})$} & \multicolumn{2}{c}{ Teste de Assimetria do Gráfico do Funil } \\
\cline { 3 - 4 } & & Alfa & IC 95\% de Alfa \\
\hline Sepse & 169 & 0,32 & $0,31-0,33$ \\
Complicações respiratórias & 169 & 1,21 & $0,11-2,31$ \\
Deiscência da anastomose & 136 & 2,70 & $1,41-3,99$ \\
Insuficiência hepática & 96 & 1,30 & $-0,79-3,39$ \\
Insuficiência renal & 96 & 0.91 & $0,90-0,92$ \\
Total & 169 & 1,78 & $0,34-3,21$ \\
\hline
\end{tabular}

IC 95\% - Intervalo de confiança de 95\%. 
to maior o valor de alfa, maior a probabilidade de estar presente viés de publicação.

Não houve restrição quanto ao idioma de publicação dos estudos. O critério mais importante para a classificação foi o sigilo de alocação, que necessariamente teve de ser mantido até o tempo da administração de corticóides. O software da colaboração Cochrane - Review Manager, versão 4.2.3 para Windows, Oxford, Reino Unido - foi empregado para metanálise dos dados ${ }^{27}$.

A avaliação de sensibilidade foi planejada para explorar fontes de heterogeneidade, quando ela existiu. $A$ heterogeneidade foi quantificada pelo teste do Quiquadrado e $\mathbf{I}^{2}$ (transformação da raiz quadrada do Quiquadrado que descreveu a proporção da variação total no estudo que é devida à heterogeneidade $)^{13}$.

Razão de chances - Odds ratio (OR) - com 95\% de confiança, foi utilizado para verificar a diferença significativa entre os grupos tratamento e placebo. O método de Peto foi utilizado para o cálculo da OR das variáveis qualitativas, porque a presença eventual de valor nulo em um estudo não afeta o cálculo e, deste modo, nenhuma aproximação tornou-se necessária ${ }^{15}$.

Foram analisados os seguintes desfechos no período pós-operatório (até trinta dias após a operação): sepse, complicações respiratórias, deiscência da ferida operatória, insuficiência hepática, insuficiência renal e mortalidade.

Nesta metanálise, o tratamento cirúrgico do câncer esofágico foi escolhido para averiguar o efeito dos corticosteróides, porque apresenta alta freqüência de complicações pós-operatórias e pela homogeneidade dos pacientes e dos locais onde foram realizados os ensaios clínicos.

Não há concordância a respeito dos benefícios dos corticosteróides no alivio do estresse cirúrgico. Esta dissensão é provavelmente causada pela variedade da dose, no tempo de administração e no tipo de fármacos utilizados, bem como a natureza dos procedimentos cirúrgicos estudados. Acresce-se a isto que os benefícios da modulação da resposta inflamatória podem se tornar inaparentes na presença de complicações ocasionadas pelas insuficiências orgânicas relacionadas com a doença de base (neoplasia) ou decorrentes do manuseio cardiopulmonar ${ }^{6,7}$. Outro fator determinante na incidência de complicações também é o número de operações realizadas por cirurgião e por hospital ${ }^{28}$.

Dentre os glicocorticóides, destacou-se o uso clínico da metilprednisolona. Estudos prévios em humanos e ratos demonstraram que a metilprednisolona parece ter concentrações maiores no pulmão que a prednisoIona $^{31}$. Quando a metilprednisolona é administrada por via venosa na dose individual de $1000 \mathrm{mg}$, a concentração máxima no sangue em adultos jovens é igual ou maior que $10 \mu \mathrm{g} / \mathrm{mL}^{12}$. A metilprednisolona pode suprimir significativamente a transformação blastóide dos linfócitos, a produção de imunoglobulinas e a atividade das células natural killers ${ }^{12}$.

Também é provável que após a esofagectomia aconteça uma resposta neurohormonal análoga ao do choque séptico, na qual vários pacientes apresentam insuficiência adrenal relativa ${ }^{32}$. Esta disfunção adrenal é temporária e após a esofagectomia, assim como no choque séptico, não foi necessária a reposição de corticosteróide em longo prazo.

A modificação causada pelo corticóide na resposta inflamatória parece ser importante num estágio inicial, porque a resposta antiinflamatória compensatória deve ocorrer numa rápida sucessão após a resposta inflamatória. Por este motivo, foram incluídos apenas estudos onde o corticosteróide foi administrado antes da agressão cirúrgica ${ }^{2,10-12}$.

\section{RESULTADOS}

A tabelas 1, 2 e 3 ilustram as características dos ensaios clínicos selecionados para metanálise: foram incluídos quatro estudos ${ }^{2,10-12}$, com o total de 169 pacientes. Destes, 82 receberam metilprednisolona (grupo tratamento) e 87 receberam solução fisiológica (grupo placebo) durante a indução anestésica. Não houve diferenças significativas nas características dos pacientes entre os grupos placebo e tratamento (Tabela 2). Foi possível verificar diferenças nos desfechos entre os grupos tratamento e placebo. Entretanto, no grupo tratamento, a variação nas doses de metilprednisolona não se correlacionou com a incidência e gravidade dos desfechos. Portanto, não foi estipulada a dose ideal de metilprednisolona a ser empregada na prevenção das complicações pós-operatórias.

A incidência de complicações pós-operatórias foi de $21 \%$ no grupo tratamento e de $66 \%$ no grupo placebo (Tabela 4). O grupo tratamento apresentou menor incidência no total de complicações, mesmo excluindo deste somatório as complicações respiratórias (Tabelas 4 e 5). Os pacientes que receberam metilprednisolona sofreram menor incidência de sepse e complicações respiratórias pós-operatórias (Tabelas 4 e 5). Nas figuras 1, 2 e 3 estão ilustradas redução significativa da 
Tabela 4 - Número de Pacientes dos Grupos Tratamento e Placebo que Apresentaram Complicações Pós-Operatórias

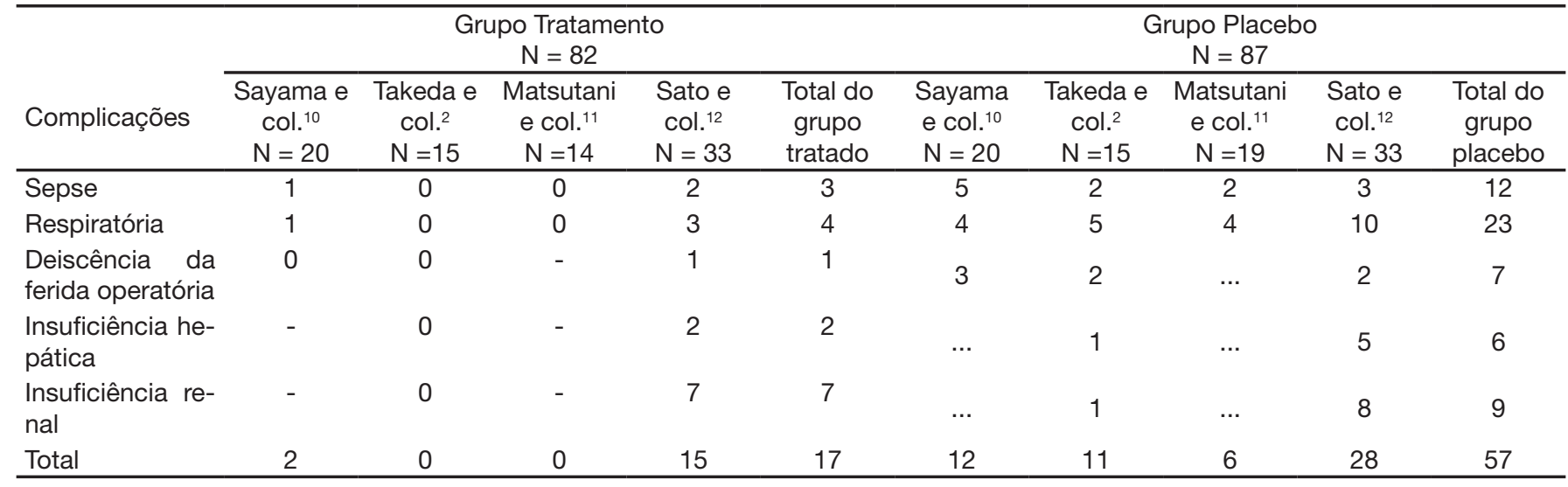

Tabela 5 - Resumo da Metanálise das Complicações Pós-Operatórias

\begin{tabular}{lccc}
\hline Desfechos Pós-Operatório & Tamanho do Efeito OR* (IC DE 95\%) & NNT & IC 95\% do NNT \\
\hline Sepse & $0,28(0,10-0,81)$ & 13 & $6-53$ \\
Complicações respiratórias & $0,20(0,09-0,46)$ & 5 & $3-8$ \\
Deiscência da anastomose & $0,23(0,05-1,11)$ & 13 & $6-387$ \\
Insuficiência hepática & $0,34(0,08-1,43)$ & 10 & $6-52$ \\
Insuficiência renal & $0,73(0,24-2,19)$ & 18 & $6-20$ \\
Total & $0,12(0,06-0,23)$ & 2 & $2-3$ \\
\hline
\end{tabular}

\section{Peto OR.}

IC 95\% -Intervalo de Confiança de 95\%.

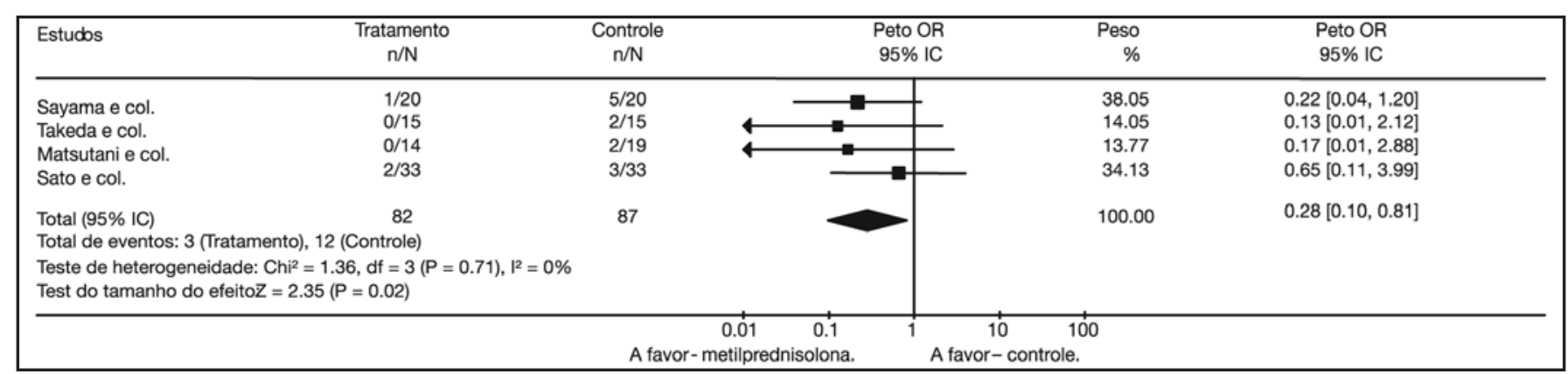

Figura 1 - Sepse pós-operatória

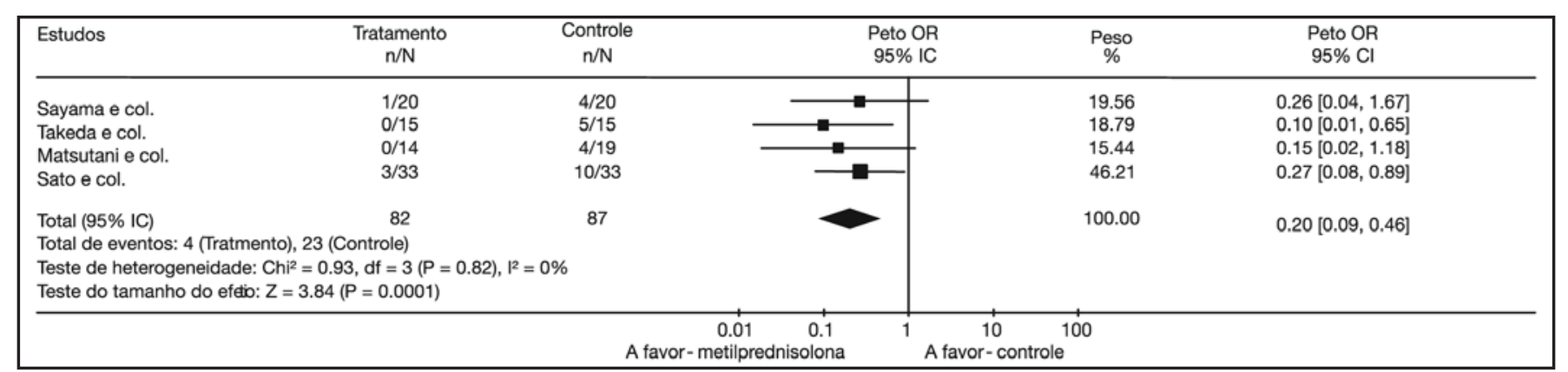

Figura 2 - Complicações Respiratórias Pós-Operatórias 


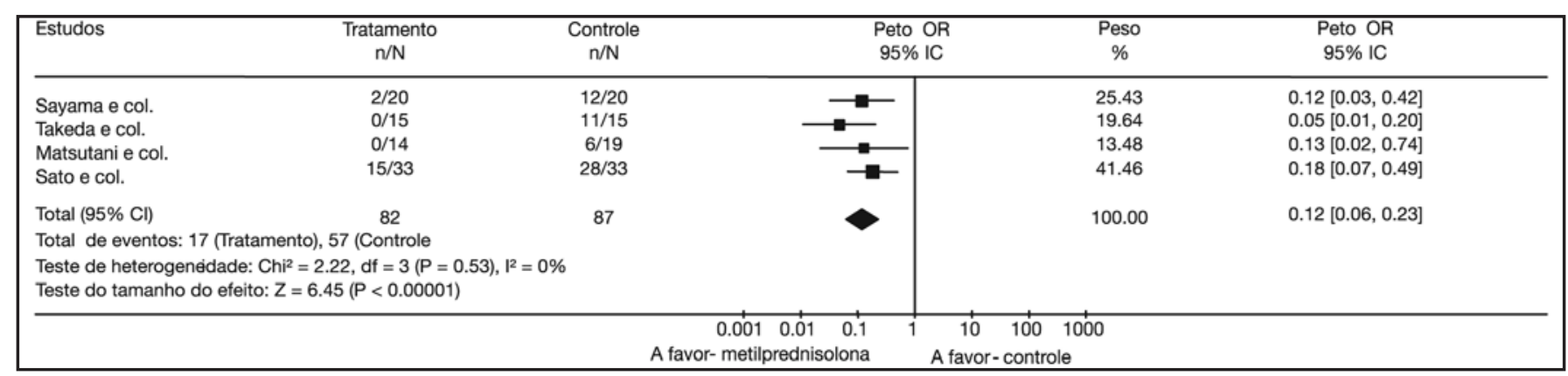

Figura 3 - Número de Complicações no Pós-Operatório

sepse, das complicações respiratórias e do total das complicações no período pós-operatório no grupo que recebeu metilprednisolona.

Ocorreram dois óbitos no grupo placebo (67 pacientes). Não ocorreram óbitos no grupo tratamento (62 pacientes). Não houve diferença de mortalidade entre os grupos tratamento e placebo, com OR $=0,13$ (IC $95 \%=0,01-2,12)$.

\section{DISCUSSÃO}

A qualidade dos artigos selecionados foi homogênea, avaliada pela escala de Jadad (Tabela 1). Diferentes sistemas de escore foram propostos por vários autores, cada um apresentando vantagens e desvantagens. Não há sistema universalmente aceito. O sistema de Jadad foi empregado nesta revisão pela sua simplicidade e por avaliar os critérios aleatórios e encobertos e perdas de cada estudo ${ }^{24}$. Não houve diferenças significativas das características dos grupos placebo e tratamento (Tabela 2).

Os desfechos que apresentaram maior assimetria em relação ao viés de publicação (Tabela 3 ) foram a deiscência da anastomose (três ensaios) e insuficiência hepática (dois ensaios). Este viés não pode ser demasiadamente valorizado, levando-se em conta o pequeno número de estudos encontrados (quatro).

A incidência de complicações pós-operatórias foi de $21 \%$ no grupo tratamento e de $66 \%$ no grupo placebo (Tabelas 4 e 5, Figura 3). Os pacientes que receberam metilprednisolona sofreram menor incidência de sepse e complicações respiratórias pós-operatórias (Tabelas 4 e 5, Figuras 1 e 2).O grupo tratamento apresentou menor incidência no total de complicações, mesmo excluindo deste somatório as complicações respiratórias (Tabelas 4 e 5).

A patogenia da disfunção orgânica pós-operatória é multifatorial, incluindo causas como hipoxemia, des- nutrição, dor, tipo de analgesia e a experiência do cirurgião ${ }^{2,10-12,32}$. A ação da metilprednisolona, ao inibir a resposta inflamatória sistêmica e regional, principalmente pulmonar ${ }^{31}$, contribuiu para a redução das complicações respiratórias, sepse e das complicações pós-operatórias como um todo.

A melhoria na sepse com o emprego da metilprednisolona pode resultar da redução do choque, da diminuição da gravidade da inflamação sistêmica, sem o incremento de outras complicações relacionadas ao uso de corticosteróides ${ }^{32}$. O mecanismo deste tipo de insuficiência adrenal na sepse é complexo e ainda não está completamente esclarecido, mas é resultado, em parte, do processo inflamatório que ocasiona a liberação inadequada de hormônio adrenocorticotrópico, em combinação com a resistência dos receptores periféricos aos esteróides. O corticosteróide se mostrou benéfico na redução da sepse, nesta metanálise, provavelmente pelo efeito cumulativo da redução das complicações respiratórias (significativo) e de outras disfunções orgânicas (não significativo).

É também plausível que casos leves de sepse possam ter sido 'mascarados' pelo uso de corticosteróides e os casos clinicamente relevantes pudessem ser semeIhantes entre os grupos. Outro fator que poderia explicar a redução da sepse pós-operatória é a escolha da metilprednisolona. Em relação a outros corticosteróides, ela apresenta algumas características que poderiam ser benéficas neste tipo de paciente: meia-vida de apenas 2,8 horas $^{12}$, concentrações maiores no pulmão que a prednisolona ${ }^{31}$ e ação antiinflamatória cinco vezes mais intensa que a do cortisol, enquanto a ação no metabolismo eletrolítico é menos da metade ${ }^{12,29,30}$.

Não houve diferença significativa na incidência pósoperatória de insuficiência hepática, insuficiência renal e deiscência de anastomose entre os grupos placebo e tratamento. O uso do corticosteróide não aumentou a hepato ou nefrotoxicidade e o processo de cicatriza- 
ção da área cirúrgica não foi afetado. Portanto, estes dados sugerem que a metilprednisolona pode ser administrada com segurança para aliviar o estresse cirúrgico.

Não houve diferença distinguível de mortalidade pósoperatória entre os grupos placebo e tratamento $\mathrm{O}$ pequeno tamanho dos ensaios clínicos estudados pode não ter proporcionado poder estatístico suficiente para detectar a diferença deste desfecho entre os grupos placebo e tratamento.

Comparando-se os desfechos dos ensaios clínicos isoladamente com os da revisão sistemática, verificou-se que a metanálise objetivamente melhorou a confiabilidade nos resultados. Isto ocorreu devido ao aumento do poder estatístico das comparações e à maior precisão da estimativa do efeito do tratamento com corticosteróide. Todos os aspectos descritos foram avaliados empregando um método, que é intrinsecamente objetivo, em oposição a opinião de experts, que é, por definição, subjetiva.

Há, entretanto, limitações em relação aos resultados do presente estudo. Fazem-se necessários estudos com um número maior de participantes para que seja possível a extrapolação segura dos resultados para a prática clínica. Outro limite da generalização dos resultados encontrados é o tipo de paciente estudado, portador de neoplasia do esôfago e submetido a ressecção do tumor, cuja debilitação no pré-operatório pode ser maior do que noutras situações de estresse cirúrgico. Questiona-se também se a administração de glicocorticóide em dose elevada, ainda que única, poderia determinar efeitos deletérios de longo prazo sobre a evolução da neoplasia (redução da sobrevida, aumento das recidivas e metástases). Deste modo, nos aspectos em que a metanálise não foi conclusiva, será possível reduzir a incerteza quando novos estudos forem acrescidos ao longo do tempo, com maior número de pacientes e tempo de acompanhamento pós-operatório prolongado (ao menos cinco ou mais anos).

\section{CONCLUSÃO}

Concluiu-se que, sobre a administração pré-operatória de metilprednisolona:

1. Ocorreu redução significativa na incidência do total de complicações pós-operatórias, sepse e complicações respiratórias no grupo que recebeu metilprednisolona em relação ao grupo placebo;

2. Não ocorreu diferença significativa entre os grupos placebo e tratamento em relação a incidência pós- operatória de óbitos, insuficiência hepática, insuficiência renal e deiscência da ferida operatória.

\section{REFERÊNCIAS}

01. Holte K, Kehlet H - Perioperative single-dose glucocorticoid administration: pathophysiologic effects and clinical implications. J Am Coll Surg, 2002;195:694-712.

02. Takeda S, Ogawa R, Nakanishi $\mathrm{K}$ et al - The effect of preoperative high dose methylprednisolone in attenuating the metabolic response after oesophageal resection. Eur J Surg, 1997;163:511-517.

03. Takeda S, Takeda S, Kim C et al - Preoperative administration of methylprednisolone attenuates cytokine-induced respiratory failure after esophageal resection. J Nippon Med Sch, 2003;70:16-20.

04. Raimondi AM - Esofagite no coto esofágico em pacientes submetidos à esofagogastroplastia para tratamento do megaesôfago avançado: avaliação clínica e endoscópica a longo prazo. (Dissertação). São Paulo: Faculdade de Medicina da Universidade de São Paulo; 2001.

05. Cecconello I, Rocha JRM, Raimondi AM et al - Reflux esophagitis and ectopic columnar epithelium in the esophageal stump after cervical gastroplasty: a reappraisal based on 17 years follow-up. Gut, 2001;49(Suppl3):3028.

06. McCulloch P, Ward J, Tekkis PP - Mortality and morbidity in gastro-oesophageal cancer surgery: initial results of ASCOT muticentre prospective cohort study. BMJ, 2003;327(7425):1192-1197.

07. Rentz J, Bull D, Harpole D et al - Transthoracic versus transhiatal esophagectomy: a prospective study of 945 patients. J Thorac Cardiovasc Surg, 2003;125:1114-1120.

08. Meduri GU, Tolley EA, Chrousos GP et a I- Prolonged methylprednisolone treatment suppresses systemic inflammation in patients with unresolving acute respiratory distress syndrome: evidence for inadequate endogenous glucocorticoid secretion and inflammation-induced immune cell resistance to glucocorticoids. Am J Respir Crit Care Med, 2002;165:983991.

09. Cooper MS, Stewart PM - Corticosteroid insufficiency in acutely ill patients. N Engl J Med, 2003:348:727-734

10. Sayama J, Shineha R, Yokota K, Ueda H, Hirayama K, Ooe H, et al - The effectiveness of preoperative steroid therapy in preventing postoperative circulatory system complications in surgery of esophageal cancer. Nippon Kyobu Geka Gakkai Zasshi, 1995;43:652-655.

11. Matsutani $T$, Onda $M$, Sasajima $K$ et al - Glucocorticoid attenuates a decrease of antithrombin III following major surgery. J Surg Res, 1998;79:158-163

12. Sato N, Koeda K, Ikeda K et al - Randomized study of the benefits of preoperative corticosteroid administration on the postoperative morbidity and cytokine response in patients undergoing surgery for esophageal cancer. Ann Surg, 2002;236:184-190.

13. Kikuchi T, Kugimiya $\mathrm{T}$ - Clinical effects of steroid in the perioperative management of patients undergoing extensive esophagectomy. Masui, 2002;51:722-727.

14. Wolf FM - Meta-Analysis: Quantitative Methods for Research Synthesis. Sage University Paper Series on Quantitative Applications in the Social Sciences. Newbury Park: Sage; 1986.

15. Leandro G - Meta-Analysis in Medical Research: the Handbook for the Understanding and Practice of Meta-Analysis. Malden: Blackwell, 2005.

16. Pearson $\mathrm{K}$ - Report on certain enteric fever inoculation statistics. BMJ, 1904;3:1243-1246.

17. Fisher RA - Statistical Methods for Research Workers. London: Oliver and Boyd; 1932.

18. Pearson $\mathrm{K}$ - On a method of determining whether a sample of size $\mathrm{n}$ supposed to have been drawn from a parent population having a known probability integral has probably been drawn at random. Biometrika, 1933;25:379-410.

19. Glass G - Primary, secondary, and meta-analysis of research. Educational Research, 1976;5:3-8

20. L'Abbe KA, Detsky AS, O'Rourke K - Meta-analysis in clinical research. Ann Intern Med, 1987;107:224-233.

21. Chalmers TC - Problems induced by meta-analyses Stat Med 1991;10:971-980.

22. Felson DT - Bias in meta-analytic research. J Clin Epidemiol, 1992;45:885- 
892.

23. Raimondi AM - Dicionário Fundamental de Bioestatística. São Paulo; 2003.

24. Jadad AR, Moore RA, Carroll D et al - Assessing the quality of reports of randomized clinical trials: is blinding necessary? Control Clin Trials, 1996;17:1-12.

25. Clarke M, Oxman AD - Cochrane reviewers' handbook 4.1.6. [updated January 2003]. In: The Cochrane Library. The Cochrane Collaboration. Oxford: Update Software; 2003.

26. Iversen GR - Bayesian Statistical Inference. Sage University Paper Series on Quantitative Applications in the Social Sciences. Newbury Park, Sage, 1984.

27. Review Manager (RevMan) [Computer program] Version 4.2.2 for Windows. Oxford, England: The Cochrane Collaboration; 2004. CD-ROM and Internet.
28. Birkmeyer JD, Stukel TA, Siewers AE et al - Surgeon volume and operative mortality in the United States. N Engl J Med, 2003:349:2117-2127.

29. Stubbs SS - Corticosteroids and bioavailability. Transplant Proc, 1975;7:11-19.

30. Fisher LE, Ludwig EA, Wald JA et al - Pharmacokinetics and pharmacodynamics of methylprednisolone when administered at 8 am versus 4 pm. Clin Pharmacol Ther, 1992;51:677-688.

31. Greos LS, Vichyanond P, Bloedow DC et al - Methylprednisolone achieves greater concentrations in the lung than prednisolone. A pharmacokinetic analysis. Am Rev Respir Dis, 1991;144(3 Pt 1):586-592.

32. Rivers EP, Mclntyre L, Morro DC et al - Early and innovative interventions for severe sepsis and septic shock: taking advantage of a window of opportunity. CMAJ, 2005;173:1054-1065. 